\title{
Laserterapia no tratamento de lesão ao nervo alveolar inferior
}

\author{
Laser therapy in the treatment of lesions to the inferior alveolar nerve \\ Terapia con láser en el tratamiento de lesiones del nervio alveolar inferior
}

Recebido: 06/06/2021 | Revisado: 12/06/2021 | Aceito: 15/06/2021 | Publicado: 30/06/2021

\author{
Carlos Eduardo de Jesus Bastos \\ ORCID: https://orcid.org/0000-0001-9894-6563 \\ Unidade de Ensino Superior de Feira de Santana, Brasil \\ E-mail: d.dubastos@gmail.com \\ Ana Viviam Souza Ferro Gomes \\ ORCID: https://orcid.org/0000-0003-3441-8205 \\ Centro Universitário Dom Bosco, Brasil \\ E-mail: anavivianfg@gmail.com \\ Thiago Freitas Leite \\ ORCID: https://orcid.org/0000-0003-3335-5951 \\ Hospital Emec, Brasil \\ Niro Health Center, Brasil \\ E-mail: cirface2011@ hotmail.com \\ Caio Cezar Rebouças e Cerqueira \\ ORCID: https://orcid.org/0000-0003-2205-5128 \\ Hospital Emec, Brasil \\ Niro Health Center, Brasil \\ E-mail: cerqueira.bucomaxilo@gmail.com \\ Lara Carolyne de Sousa Flor \\ ORCID: https://orcid.org/0000-0002-5525-4572 \\ Centro Universitário Dom Bosco, Brasil \\ E-mail: laracarolynef@gmail.com \\ José Manuel Noguera Bazán \\ ORCID: https://orcid.org/0000-0002-1700-8995 \\ Centro Universitário Dom Bosco, Brasil \\ E-mail: jmnbazan@hotmail.com
}

\begin{abstract}
Resumo
Danos ao nervo alveolar inferior consistem em uma das complicações mais comuns nas exodontias de terceiros molares inclusos. Lesões nervosas podem acarretar em hiperalgia refratária, parestesia e prurido. Um planejamento minucioso, por meio de exames de imagens, como a tomografia computadorizada, além do conhecimento anatômico do nervo e manejo cuidadoso dos tecidos, é fundamental para evitar complicações. O diagnóstico do deficit neural emprega métodos subjetivos por meio de questionários e exame físico ou mais objetivamente com exames apurados de condução do impulso nervoso. O tratamento é desafiador, diversas modalidades cirúrgica, medicamentosa e clínica são aplicadas, dentre elas a aplicação do laser de baixa intensidade. O objetivo deste trabalho é discorrer sobre a efetividade da aplicação do laser mediante revisão da literatura do estado da arte. Foi realizada pesquisa nas bases de dados Medline, Pubmed e Google Acadêmico, sendo selecionados a partir dos critérios de inclusão e exclusão determinados.
\end{abstract}

Palavras-chave: Nervo mandibular; Parestesia; Terapia a laser.

\begin{abstract}
Inferior alveolar nerve damage is one of the most common complications in impacted third molar extractions. Nerve damage can lead to refractory hyperalgia, paresthesia and itching. Careful planning, through imaging exams such as computed tomography, in addition to anatomical knowledge of the nerve and careful tissue management, is essential to avoid complications. The diagnosis of neural deficit employs subjective methods through questionnaires and physical examination or more objectively with accurate examinations of nervous impulse conduction. The treatment is challenging, several surgical, drug and clinical modalities are applied, including the application of low-intensity laser. The objective of this work is to discuss the effectiveness of laser application through a review of the state-of-the-art literature. A search was performed in Medline, Pubmed and Academic Google databases, being selected based on the determined inclusion and exclusion criteria.
\end{abstract}

Keywords: Mandibular nerve; Paresthesia; Laser therapy.

\section{Resumen}

El daño del nervio alveolar inferior es una de las complicaciones más comunes en las extracciones de terceros molares impactados. El daño a los nervios puede provocar hiperalgia refractaria, parestesia y picazón. Una planificación 
cuidadosa, a través de pruebas de imagen como la tomografía computarizada, además del conocimiento anatómico del nervio y el manejo cuidadoso de los tejidos, es esencial para evitar complicaciones. El diagnóstico de déficit neural emplea métodos subjetivos a través de cuestionarios y examen físico o de manera más objetiva con exámenes precisos de la conducción del impulso nervioso. El tratamiento es desafiante, se aplican varias modalidades quirúrgicas, farmacológicas y clínicas, incluida la aplicación de láser de baja intensidad. El objetivo de este trabajo es discutir la efectividad de la aplicación del láser a través de una revisión de la literatura más actualizada. Se realizó una búsqueda en las bases de datos Medline, Pubmed y Academic Google, siendo seleccionada en base a los criterios de inclusión y exclusión determinados.

Palabras clave: Nervio mandibular; Parestesia; Terapia por láser.

\section{Introdução}

Lesão ao nervo alveolar inferior (NAI) decorrente do ato cirúrgico é uma das principais complicações associadas às cirurgias de terceiros molares inferiores e de instalação de implantes (Lopes \& Freitas, 2013). Esta lesão pode levar à uma parestesia do nervo, promovendo desconforto e incômodo ao paciente, principalmente ao realizar atividades rotineiras e sociais, podendo interferir na fonação, alimentação, e relacionamentos afetivos (Dodo, Sotto-Maior, Faot, Del Bel Cury, \& Senna, 2015).

$\mathrm{Na}$ literatura as alterações sensoriais referidas pelos pacientes são das mais variadas, muitos alegam sensação de inchaço, dormência, formigamento, dores intermitentes como alfinetadas, agulhadas, e até mesmo sensação de choque elétrico. Outro nervo que também pode ser afetado na cirurgia de terceiros molares inclusos é o nervo lingual (NL). Pode acarretar parestesia ipsilateral dos dois terços anteriores da língua, mucosa do assoalho bucal e gengiva lingual (Ramadorai, Tay, Vasanthakumar, Lye, \& surgery, 2019). Estes sintomas podem ser transitórios ou permanentes, variam conforme o grau de severidade do dano nervoso (Misch \& Resnik, 2010).

A lesão ao NAI acontece na maioria dos casos pelo planejamento inadequado, visto que este acidente ocorre em razão da proximidade do NAI com a sítio cirúrgico, o que pode ser prevenido principalmente através de recursos imaginológicos, como a tomografia computadorizada de feixe cônico, que permite uma avaliação tridimensional além de visualizar por meios de cortes parassagitais a relação existente entre a unidade dentária a ser extraída e o canal mandibular (Shavit \& Juodzbalys, 2014).

O diagnóstico das disfunções nervosas é feito inicialmente por meio dos relatos subjetivos do paciente, após cessado efeito da anestesia é relatado a perenicidade da sensação de dormência quando o paciente retorna para avaliação pósoperatória. Existem ainda métodos mecanoceptivos, nociceptivos e exames específicos para investigar a condução nervosa local. Existem diversos tratamentos descritos desde anastomoses cirúrgicas, tratamentos medicamentosos com polivitamínicos e estimuladores neurais até métodos físicos como a laserterapia de baixa potência (Juodzbalys, Wang, \& Sabalys, 2011).

A terapia a laser de baixa intensidade ou low-level laser terapy (LLLT) é utilizada comumente na área de fisioterapia (Campos et al., 2021). O LLLT pode acelerar a cicatrização de feridas, minimizar sintomatologia dolorosa, restaurar a função neural, melhorar a cicatrização, dentre outros benefícios (Pol et al., 2016; Rodrigues et al., 2021). Desta forma, este trabalho objetiva verificar a efetividade do laser de baixa intensidade para o tratamento de danos ao nervo alveolar inferior referentes a complicações pós-cirúrgicas em cirurgia oral menor.

\section{Metodologia}

Trata-se de uma pesquisa qualitativa, caracterizada por uma revisão de literatura acerca do tema escolhido. Na qual as etapas posteriores foram por meio da definição do tema abordado, seleção dos critérios de inclusão e exclusão, levantamento bibliográfico conforme as bases de dados selecionadas e síntese na escrita dessa revião. Conforme Pereira, Shitsuka, Parreira, and Shitsuka (2018) existem aspectos metodológicos no estudo qualitativo que possibilitam ao pesquisador, mostrar suas próprias concepções referentes ao objeto estudado. 
A literatura foi selecionada no período de maio de 2021 nas bases de dados: Scientific Electronic Library Online Brasil (SCIELO/ BR); Literatura Latino-Americana e do Caribe em Ciências da Saúde (LILACS); Medical Literature Analysis and Retrieval System Online (MEDLINE) e Biblioteca Virtual em Saúde (BVS). Quanto aos critérios de inclusão, foram pesquisados artigos publicados em inglês, português ou espanhol, no período de 2011 a 2021. Revisões de literatura, relatos de caso e ensaios clínicos randomizados. Foram excluídos trabalhos de conclusão de cursos, monografias, estudos clínicos em animais, capítulos de livros, textos publicados em anais congressos e ainda, trabalhos anteriores a 2011.

As palavras-chave são descritores em Ciências da Saúde (DECs): Nervo Mandibular (Mandibular Nerve), Parestesia (Paresthesia) e Terapia a Laser (Laser Therapy). Inicialmente a seleção dos artigos foi a partir dos descritores nas bases de dados referidas, após da seleção destes através dos critérios de inclusão, foi realizada a leitura do título, do resumo e por fim, do artigo na íntegra para integra-los nesta pesquisa.

\section{Resultados e Discussão}

A busca online resultou em um total de 420 artigos pelo Google Acadêmico através da busca de todos os descritores, enquanto no Pubmed foi obtido 5 artigos disponíveis, 5 trabalhos foram encontrados na plataforma Medline, 2 na BBO, 2 na IBCES e 2 na LILACS. Dentre os quais após aplicação de todos os filtros, leitura dos títulos, leitura do resumo e por fim, do artigo na íntegra pode-se adotar o total de 20 trabalhos para fundamentar esta pesquisa.

Para Benevides, Valadas, Diógenes, \& Rodrigues (2018), é possível associar a ocorrência da parestesia do nervo alveolar inferior com procedimentos cirúrgicos. Uma vez que, evidenciraram em seus estudos fatores como o tipo de incisão utilizada, curetagem vigorosa no interior do alvéolo sem uma visualização do feixe vásculo-nervoso, hemorragias e profundidade do implante sem um planejamento adequado são alguns exemplos favoráveis para ocasionar esse tipo de lesão. Assim, como complementado por Coulthard et al. (2014) que tanto o nervo alveolar inferior quanto lingual, podem ser lesionados em vários procedimentos, seja durante a anestesia local, cirurgia ortognática, trauma, cirurgia de terceiro molar, entubação e acesso submandibular. Principalmente, em cirurgias de terceiros molares, na qual Hakimiha et al. (2020) afirma que é de $43 \%$ entre os procedimentos orais menores.

Contudo, Lopes \& Freitas (2013) afirmam que a etiologia da parestesia pode originar-se de fatores diretos ou indiretos, tanto locais quanto sistêmicos. E que a perda da sensibilidade do nervo afetado promove um desconforto de forma transitória ou permanente em ambas situações, dependendo da injúria e intensidade. Hakimiha et al. (2020) cita que os principais sinais e sintomas são a hipoesteria, paretestesia, alodinia, anestesia do lábio, dentes e mucosa referentes ao nervo afetado. Assim, afetando diretamente a vida do paciente, de forma que implica nas suas relações sociais de forma negativa. Dessa forma, várias modalidades de tratamento podem ser empregadas, tais como a microneurocirurgia, indicada principalmente para casos de neurotmnese (Hakimiha et al., 2020).

Segundo Girão Evangelista, Pontes Tabosa, Bezerra, and de Araújo Neto (2019) uma modalidade bastante estudada é o tratamento com laserterapia de baixa intensidade, que consiste numa abordagem não invasiva que consegue agir em moderadas lesões ao NAI. O tratamento com LLLT evidenciado por Santos et al. (2019) consiste na utilização de luz baixa (menor que 100-200 mW). Este método apresenta efeito estimulante nos tecidos-alvo, sendo empregado a fim de minimizar a dor, inflamação, estimular o colágeno e cicatrização tecidual. Conforme Miloro \& Criddle (2018), a avaliação da eficácia da aplicação de laser de baixa intensidade precisa a princípio de um método adequado de estimativa do dano nervoso ao NAI. Ao passo que, uma das dificuldades deste mesmo estudo foi encontrar testes uniformes e um consenso quanto a gravidade de dano ao NAI.

Mas, Seguro \& Oliveira (2014) ja haviam realizado um estudo sobre os testes neurossensoriais que são feitos para determinar o grau de lesão ao nervo fundamentado em toques estáticos, que por sua vez o paciente indica a diferença entre dois 
pontos com discrepâncias quanto a sensibilidade, e através de meios nociceptivos, realizados com testes térmicos e dor (utilizase uma agulha).

Assim, simultaneamente ao estudo de Miloro \& Criddle 2018, houve uma pesquisa que afirmou a influência do LLLT em melhorar a alteração neurossensorial, uma vez que ela é útil também na cicatrização mais rápida do dano causado no nervo, possibilitando mais bem-estar dos pacientes em função da melhora significativa da dor (Esmaeelinejad \& Motamedi, 2018). Compativel com o estudo clássico de Khullar, Brodin, Barkvoll, and Haanaes (1996) que realizou uma pesquisa com 13 pacientes após o uso da terapia a laser de baixa intensidade obteve melhorias na percepção sensorial mecânica.

Todavia, em um estudo randomizado foi obtido um valor expressivo de, aproximadamente $80 \%$ dos pacientes tratados com recuperação neurossensorial a partir dos testes objetivos e subjetivos. Constatando que há uma melhora neurossensorial significativa na terapia com LLLT, mas nas pesquisas apresentadas, os autores não expuseram o tempo de tratamento dos pacientes. Assim, como refletido por Neto e Catão, essas informações são cruciais também para que haja a replicação do estudo futuramente (Pol et al., 2016).

Oliveira e associados (2015) executaram um estudo retrospectivo em 125 pacientes, onde afirmaram que outros fatores também interferem na recuperação do paciente, tais como idade, intervalo entre o tempo cirúrgico e início da terapia com o LLLT (de Oliveira et al., 2015).

Apesar disso, é demonstrado em uma pesquisa realizada por Grillo, Wada e Sousa (2014) uma recuperação total da sensibilidade do nervo alveolar inferior entre $54 \%$ e $83 \%$ dos pacientes a partir da $6^{\circ}$ sessão do uso do LLLT, que resulta na eficiência desse tipo de tratamento, apesar de não ter relatado mais detalhadamente os passos seguidos para tal recuperação (Grillo, Wada, \& de Sousa, 2014). Ademais, citando como caso analógo um estudo realizado por de Oliveira, da Silva, Simoes, Youssef, \& de Freitas (2015), foi apresentado excelentes resultados referentes ao uso do LLLT para o tratamento de parestesia no NAI, até mesmo em casos que possuíam maior tempo de diagnóstico.

Do mesmo modo, comprovado através de um estudo realizado por Gasperini e colaboradores em 2014, que a utilização do LLLT entre $5 \mathrm{~J} / \mathrm{cm}^{2}$ para exposição intra-oral e $30 \mathrm{~J} / \mathrm{cm}^{2}$ ou $70 \mathrm{~J} / \mathrm{cm}^{2}$ para extra oral densidade de energia possui importantes ações anti-inflamatórias e analgésicas, pois reduziu significativamente o incomodo no lado irradiado no período de 24 e 72 horas nas avaliações pós-operatórias. Uma semana depois, nenhum paciente relatou dor nos dois lados (Gasperini, Siqueira, \& Costa, 2014). Pode-se comparar a eficácia do laser em lesão ao NAI, também a casos de osteoradionecrose, que representa um complexo tratamento, mas a literatura dispõe de casos de sucesso associados ao uso do laser de baixa intensidade (da Silva-Souza et al., 2021).

Porém, para Carroll, Milward, Cooper, Hadis, \& Palin (2014), a aplicação do LLLT só é possível ser realizada por meios ativos do diodo que possuem comprimentos de onda vermelhos de 632nm, 660nm atuando nas camadas mais superficiais dos tecidos e infravermelhos 820,940nm, que agem nas regiões mais profundas. Mas um ano depois, foi evidenciado na literatura diferentes níveis de comprimentos quanto a irradiação e dosimetria usada, o que torna difícil obter uma informação esclarecedora e concreta quanto a aplicação clinica pelo profissional, dessa forma, conclui-se que não há um padrão (de Oliveira et al., 2015).

É possível citar ainda o estudo clínico duplo cego randomizado pelos autores Miloro e Criddle (2018), em que a amostra do estudo apresentava 35 pacientes com lesão iatrogênica, dentre as variáveis do estudo o nervo afetado (lingual ou alveolar inferior) foram selecionados e o tempo passado desde a lesão até o tratamento, diferenciando entre 3 a 12 meses ou maior que 12 meses. Em ambos os nervos envolvidos houveram uma melhora de 46\% no grupo de LLLT em comparação com a melhora de $38 \%$ no grupo controle. Os autores não notaram diferença no tocante tempo, do período da lesão até o tratamento. existindo a necessidade de maiores estudos clínicos sobre esta temática, em virtude da escassez deste conteúdo (Miloro \& Criddle, 2018). 
Em relação a localização do laser para atingir os nervo alveolar inferior, bem como suas ramificações, é preciso operar no forame mandibular e forame mentoniano de forma que, as aplicações precisarão ser repetidas em intervalos de 48 horas até que o sintoma desapareça, em todo o comprimento (Neto \& de Vasconcelos Catão, 2020).

\section{Conclusão}

Foi evidenciado neste trabalho que o laser de baixa intensidade apresenta melhora significativa na atividade neurossensorial do nervo alveolar inferior. Não obstante, alguns impasses foram observados, tais como um protocolo padrão de tratamento para que seja possível a sua replicação posterior.

Ainda, alguns autores apresentaram diferentes pontos de vista quanto ao tempo de lesão até o tratamento, em alguns ensaios clínicos não obtiveram diferença entre os grupos com o período da lesão até o tratamento. Enquanto outros relataram que fatores importantes como a idade do paciente e o período mais curto entre a lesão e o tratamento.

Portanto é primordial que trabalhos futuros abordem numa especificação de um protocolo padrão, para a segurança em aplicação nos pacientes frente à obtenção de resultados. Assim, é necessário que haja mais pesquisas que esclareça tais informações.

\section{Referências}

Campos, L., Martins, F., de Macedo, D. S., Sendyk, W. R., Tanaka, M. H. J. R., Society, \& Development. (2021). Laser de alta potência para cirurgia periodontal em paciente com síndrome de Down: Relato de caso. 10(6), e30110615834-e30110615834.

Coulthard, P., Kushnerev, E., Yates, J. M., Walsh, T., Patel, N., Bailey, E., \& Renton, T. F. (2014). Interventions for iatrogenic inferior alveolar and lingual nerve injury. Cochrane Database Syst Rev(4), Cd005293. doi:10.1002/14651858.CD005293.pub2

Esmaeelinejad, M., \& Motamedi, M. H. K. (2018). Effect of low-level laser on the healing of neurosensory disturbance following sagittal split ramus osteotomy: a double-blind, randomized clinical trial. J Iranian Red Crescent Medical Journal, 20(12).

Gasperini, G., Siqueira, L., \& Costa, L. (2014). Terapia a laser de baixa potência na melhora de distúrbios neuro-sensoriais resultantes de osteotomia mandibular sagital bilateral: Um ensaio clínico cruzado randomizado. J Craniomaxillofac Surg, 42, 130-133.

Girão Evangelista, Í., Pontes Tabosa, F. B., Bezerra, A. V., \& de Araújo Neto, E. V., Jr. (2019). Low-Level Laser Therapy in the Treatment of Inferior Alveolar Nerve Paresthesia After Surgical Exeresis of a Complex Odontoma. J Lasers Med Sci, 10(4), 342-345. doi:10.15171/jlms.2019.55

Grillo, C. M., Wada, R. S., \& de Sousa, M. d. L. R. (2014). Acupuncture in the management of acute dental pain. J Journal of acupuncture meridian studies, $7(2), 65-70$.

Hakimiha, N., Dehghan, M. M., Manaheji, H., Zaringhalam, J., Farzad-Mohajeri, S., Fekrazad, R., \& Moslemi, N. (2020). Recovery of inferior alveolar nerve by photobiomodulation therapy using two laser wavelengths: A behavioral and immunological study in rat. J Photochem Photobiol B, 204 , 111785 . doi:10.1016/j.jphotobiol.2020.111785

Juodzbalys, G., Wang, H.-L., \& Sabalys, G. (2011). Injury of the inferior alveolar nerve during implant placement: a literature review. 2(1).

Khullar, S. M., Brodin, P., Barkvoll, P., \& Haanaes, H. R. (1996). Preliminary study of low-level laser for treatment of long-standing sensory aberrations in the inferior alveolar nerve. J Oral Maxillofac Surg, 54(1), 2-7; discussion 7-8. doi:10.1016/s0278-2391(96)90290-6

Lopes, G. B., \& Freitas, J. J. A. B. O. (2013). Parestesia do nervo alveolar inferior após exodontia de terceiros molares. 9(2), 35-40.

Miloro, M., \& Criddle, T.-R. (2018). Does low-level laser therapy affect recovery of lingual and inferior alveolar nerve injuries? Journal of Oral Maxillofacial Surgery, 76(12), 2669-2675.

Misch, C. E., \& Resnik, R. J. I. d. (2010). Mandibular nerve neurosensory impairment after dental implant surgery: management and protocol. 19(5), 378-386.

Neto, J. d. A. F., \& de Vasconcelos Catão, M. H. C. (2020). Laser Therapy in the Treatment of Patients with Oral Paresthesia: a review of clinical trials. $J$ Journal of Health Sciences, 22(1), 7-13.

Oliveira, R. F., de Andrade Salgado, D. M. R., Trevelin, L. T., Lopes, R. M., da Cunha, S. R. B., Aranha, A. C. C., . . de Freitas, P. M. (2015). Benefits of laser phototherapy on nerve repair. J Lasers in medical science, 30(4), 1395-1406.

Dodo, C. G., Sotto-Maior, B. S., Faot, F., Del Bel Cury, A., \& Senna, P. M. J. D. P. I. (2015). Lesão do nervo alveolar inferior por implantes dentários: prevenção, diagnóstico e tratamento. 9(4), 57-66.

Pereira, A. S., Shitsuka, D. M., Parreira, F. J., \& Shitsuka, R. (2018). Metodologia da pesquisa científica.

Pol, R., Gallesio, G., Riso, M., Ruggiero, T., Scarano, A., Mortellaro, C., \& Mozzati, M. J. J. o. C. S. (2016). Effects of superpulsed, low-level laser therapy on neurosensory recovery of the inferior alveolar nerve. 27(5), 1215-1219. 
Research, Society and Development, v. 10, n. 7, e50110716881, 2021

(CC BY 4.0) | ISSN 2525-3409 | DOI: http://dx.doi.org/10.33448/rsd-v10i7.16881

Ramadorai, A., Tay, A. B., Vasanthakumar, G., Lye, W. J. J. o. m., \& surgery, o. (2019). Nerve injury after surgical excision of mandibular third molars under local anesthesia: an audit. 18(2), 307-313.

Rodrigues, B. A. L., de Carvalho, A. L. V., de Melo, L. S. A., da Silva, L. R. G., da Silva, E. L. M. S. J. R., Research, Society, \& Development. (2021). Tipos de Lasers e suas aplicações em Odontopediatria. 10(5), e31810514963-e31810514963.

Shavit, I., \& Juodzbalys, G. (2014). Inferior alveolar nerve injuries following implant placement-importance of early diagnosis and treatment: a systematic review. 5(4).

Silva-Souza, L. G., de Oliveira, L. D., Nunes, G. P., dos Santos Cividanes, L., Dahan, C. M., Pereira, A. K. G., . . Development. (2021). Effectiveness of the low-level laser therapy in the management of bisphosphonate-induced osteonecrosis of the jaws: A case report. 10(6), e17510615199-e17510615199. 